\title{
Mängelansprüche bei fehlerhafter Herkunftszuordnung eines Kunstwerks in einem Auktionskatalog
}

\author{
OLG Frankfurt a.M. Urteil vom 3.5.2018 - 19 U 188/15
}

ECLI:DE:OLGHE:2018:0503.19U188.15.00

\section{Amtliche Leitsätze:}

1. Eine Zeichnung, die entgegen der vom Verkäufer erstellten Katalogbeschreibung nicht der Hand des konkret benannten Künstlers zuzuordnen ist, ist mangelhaft.

2. Ein Verkäufer, der sich hinsichtlich der Herkunftszuordnung entgegen einer schriftlich publizierten Einschätzung eines Experten auf mündliche Angaben anderer Sachverständiger verlässt, handelt arglistig im Rechtssinne, wenn er die Herkunftszuordnung des Experten in seiner Katalogbeschreibung ohne Einschränkung als, ,fälschlich zugeschrieben' bezeichnet, ohne die ihm zugetragenen gegenteiligen mündlichen Angaben hinlänglich kritisch überprüft zu haben.

\section{Vorinstanz:}

LG Frankfurt a.M. - 30.7.2017 - 2-26 O 349/14

\section{Tenor:}

Auf die Berufung des Klägers wird das am 30.7.2015 verkündete Urteil der 26. Zivilkammer des Landgerichts Frankfurt a.M. abgeändert und wie folgt neu gefasst:

1. Die Beklagte wird verurteilt, an den Kläger $22.000,00 €$ nebst Zinsen in Höhe von 5 Prozentpunkten über dem jeweiligen Basiszinssatz p.a. seit dem 1.5.2014 zu zahlen,

Zug um Zug gegen Rückgabe und Rückübereignung der im Jahre 2008 gemäß „Rechnung/Lieferschein Nr. ..." als Werk Carl Philipp Fohrs verkauften Zeichnung „Bildtitel1", "Tuschfederzeichnung in Grauschwarz und Grau über Bleistift 1812".

2. Es wird festgestellt, dass sich die Beklagte mit der Rücknahme der in Ziffer 1. bezeichneten Zeichnung in Annahmeverzug befindet.

Die Kosten des Rechtsstreits hat die Beklagte zu tragen.

Das Urteil des Senats ist vorläufig vollstreckbar. Die Beklagte darf die Vollstreckung gegen Sicherheitsleistung in Höhe von $120 \%$ des vollstreckbaren Betrags abwenden, wenn nicht der Kläger vor der Vollstreckung Sicherheit in Höhe von $120 \%$ des jeweils zu vollstreckenden Betrags leistet.

Der Streitwert wird für die Berufungsinstanz auf 22.000,00€ festgesetzt.

\section{Gründe}

I.

Die Parteien streiten über die Rückabwicklung eines Kaufvertrages über den Erwerb einer Tuschfederzeichnung aus dem beginnenden 19. Jahrhundert, deren Urheberschaft zwischen den Parteien im Streit steht.

Wegen des erstinstanzlichen Sach- und Streitstands und der dort gestellten Anträge wird auf die tatsächlichen Feststellungen im angefochtenen Urteil (BI. 216 ff. d.A.) Bezug genommen, die dahin ergänzt werden, dass im Katalog der Beklagten folgende Bestimmungen abgedruckt waren:

Sämtliche im Katalog aufgeführten Zeichnungen sind verkäuflich. Preise auf Anfrage. Reservierungen sind grundsätzlich möglich, jedoch nicht länger als drei Arbeitstage. Festbestellungen haben stets Vorrang. Der Verkaufspreis ist sofort fällig und beinhaltet die gesetzliche Mehrwertsteuer. Der Versand erfolgt auf eigene Gefahr und Kosten des Bestellers. Eigentumsvorbehalt gemäß § 449 BGB. Die Katalogbeschreibungen erfolgten nach bestem Wissen und Gewissen, sie sind keine Garantien im Rechtssinne. Der Erhaltungszustand der einzelnen Blätter ist, falls nicht anders vermerkt, gut. Die Maßangaben beziehen sich auf die Blattgröße bzw. Größe der Umrandung. Die Höhe wird von der Breite angegeben. Die Blätter liegen unter Passepartout. Erfüllungsort und Gerichtsstand ist Stadt1.

Das Landgericht hat die Klage abgewiesen. Ein Anspruch aus Verschulden bei Vertragsschluss sei wegen des Vorrangs der kaufrechtlichen Gewährleistungsvorschriften ab Gefahrübergang ausgeschlossen, soweit der Verkäufer nicht arglistig gehandelt habe, was der Kläger im vorliegenden Fall nicht bewiesen habe. Ob die streitgegenständliche Zeichnung tatsächlich von Carl Philipp Fohr stamme, könne dahinstehen. Denn der Kläger habe nicht bewiesen, dass der Geschäftsführer der Beklagten zum Zeitpunkt des Verkaufs insoweit nicht in gutem 
Glauben gewesen sei. Vielmehr habe dieser im Rahmen seiner mündlichen Anhörung glaubhaft dargelegt, auf die von mehreren Experten bestätigte Urheberschaft Carl Philipp Fohrs vertraut zu haben.

Ebenso wenig könne davon ausgegangen werden, der Geschäftsführer der Beklagten habe eine möglicherweise unrichtige Behauptung ohne tatsächliche Grundlage ins Blaue hinein aufgestellt. Ob sich zum Zeitpunkt des Verkaufs unter Kunsthistorikern eine abweichende herrschende Meinung herausgebildet habe, sei unerheblich, da dies allenfalls den Vorwurf von Fahrlässigkeit begründen könne, was für die Annahme arglistigen Verhaltens nicht genüge.

Eine auf Verschweigen von Tatsachen gestützte Anfechtung des Kaufvertrages komme gleichermaßen nicht in Betracht. Denn der Kläger habe auch nicht beweisen können, dass dem Geschäftsführer der Beklagten eine tatsächlich nicht gegebene Urheberschaft Fohrs als offenbarungspflichtige Tatsache bekannt gewesen sei. Eine solche folge insbesondere nicht aus den Aufsätzen, die der Kläger vorgelegt habe. Im Rahmen seiner Anhörung habe der Geschäftsführer der Beklagten vielmehr schlüssig und glaubhaft erklärt, nach seiner Auffassung behandelten beide Aufsätze die Frage einer Händescheidung zwischen den Künstlern Fohr und Rottmann lediglich in abstrakter Weise, ohne sich konkret auch mit der streitgegenständlichen Zeichnung zu beschäftigen. Deshalb könne auch dahinstehen, ob der Aufsatz SV1s dem Geschäftsführer der Beklagten zum Verkaufszeitpunkt bekannt gewesen sei oder nicht.

Ob ein Verkäufer aus Verschulden bei Vertragsschluss auch dann hafte, wenn er die Verpflichtung übernommen habe, den Käufer hinsichtlich von Eigenschaften der Kaufsache bzw. anderer für den Kauf relevanter Umstände zu beraten, könne auf sich beruhen, da die Beklagte derartige Beratungspflichten nicht übernommen habe. Keine der von der Rechtsprechung hierzu entwickelten Fallgruppen sei für die vorliegende Konstellation einschlägig, darüber hinaus sei aber auch gar nicht zu ersehen, dass der Kläger besonders schutzwürdig sei, da er selbst über ein erhebliches Maß an entsprechender Sachkunde verfüge.

Wegen der weiteren Einzelheiten wird auf die Gründe der angefochtenen Entscheidung (BI. 229 ff. d.A.) Bezug genommen.

\section{II.}

Gegen das ihm am 11.8.2015 (BI. 234 d.A.) zugestellte Urteil hat der Kläger am 3.9.2015 Berufung eingelegt (BI. 256 f. d.A.) und diese durch am 11.11.2015 eingegangenen Schriftsatz (BI. 270 ff. d.A.) binnen verlängerter Frist (Bl. 268 d.A.) begründet. Mit seinem Rechtsmittel verfolgt der Kläger sein Klagebegehren unverändert weiter.
1. Das Landgericht verkenne zunächst, dass die Haftung für vorsätzliches Verhalten nicht die einzige Ausnahme von dem Grundsatz sei, dass eine Haftung für Verschulden bei Vertragsschluss bei Kaufverträgen nach dem Gefahrübergang ausgeschlossen sei. Im Rahmen seiner Prüfung arglistiger Täuschung habe das Landgericht die Anforderungen an den Beweis für eine solche Täuschung überzogen und sei von nicht zutreffenden rechtlichen Annahmen ausgegangen. Zwar müsse der Käufer sämtliche Umstände, die den Arglisttatbestand ausfüllten, darlegen und beweisen, jedoch griffen zu seinen Gunsten die Erleichterungen für den Beweis von Negativtatsachen ein. Den Verkäufer treffe die sekundäre Darlegungslast, eine angeblich erfolgte Aufklärung in räumlicher, zeitlicher und inhaltlicher Weise zu spezifizieren; nur das Nichtvorliegen gerade dieser konkreten Umstände müsse der Käufer beweisen.

Nicht tragfähig sei es daher, wenn das Landgericht meine, der Kläger habe nicht beweisen können, dass der Geschäftsführer der Beklagten bezüglich der Urheberschaft Fohrs nicht in gutem Glauben gewesen sei. Ebenso wenig komme es darauf an, dass der Geschäftsführer der Beklagten vor dem Landgericht geäußert habe, er habe auf die angeblich von mehreren Experten bestätigte Urheberschaft Fohrs vertraut. Aus eigener Kenntnis habe der Geschäftsführer der Beklagten hierzu nämlich nichts gewusst. Er habe nur von Dritten gehört, dass er auf die Urheberschaft Fohrs vertrauen dürfe. Der Kläger hingegen habe dargelegt und unter Beweis gestellt, dass es zum Zeitpunkt des Verkaufs definitiv keine von mehreren Experten bestätigte Urheberschaft Carl Philipp Fohrs gegeben habe. Im Gegenteil sei dem Geschäftsführer der Beklagten unstreitig bekannt gewesen, dass SV2 als der seinerzeit ausgewiesene Experte für das Werk Fohrs das Blatt Rottmann zugeordnet habe. Schon hieraus ergebe sich die Bösgläubigkeit des Geschäftsführers der Beklagten.

Soweit das Landgericht der Beklagten zugutehalte, vor dem Verkauf die Einschätzung von Fachleuten eingeholt zu haben, übergehe es, dass der Geschäftsführer der Beklagten die Stellungnahmen SV1s und SV2s nur vom Hörensagen gekannt habe, und verkenne zugleich, dass SV2s Katalogbeitrag von 1997/98 dem diametral entgegenstehe. Zudem stelle es unbewiesene mündliche Äußerungen SV2s und SV1s über die schriftlichen Ausführungen beider Experten. SV1 habe in seinem Schreiben an den Kläger zudem klar ausgeführt, dass er an die ihm zugeschriebene mündliche Einordnung keinerlei Erinnerung habe. Das Mindeste, was die Beklagte nach Auffassung des Klägers geschuldet habe, sei dann aber eine Rücksprache mit SV2 gewesen, um den Widerspruch zwischen der angeblich mündlich mitgeteilten Einordnung des Werkes und dem schriftlichen Katalogbeitrag aufzuklären - was unstreitig nie erfolgt sei. Dies wiege umso schwerer, als der Geschäftsführer der Beklagten eigenen Angaben zufolge mit SV2 in gutem regelmäßigem Kontakt gestanden habe. 
In ihrem Verkaufskatalog habe die Beklagte dann aber weder offengelegt, dass die "fälschliche" Zuschreibung des Werks zu Rottmann durch SV2 erfolgt sei, noch, dass sie diesen niemals wegen dessen angeblichen Fehlers konsultiert habe. Ebenfalls nicht offengelegt habe sie die ihr bekannten Ausführungen SV1s aus dessen Aufsatz von 2001 über die Motivgleichheit in den Jugendwerken Fohrs und Rottmanns. Die Fachwelt, namentlich die ausgewiesenen Experten SV2 und SV1, habe 2008 nicht an der Urheberschaft Rottmanns gezweifelt, sondern sei von ihr überzeugt gewesen; das habe der Geschäftsführer der Beklagten auch gewusst und dem Kläger gleichermaßen verschwiegen.

Was ihre Gutgläubigkeit angehe, sei es Sache der Beklagten gewesen, darzulegen und zu beweisen, aufgrund welcher Umstände sie davon ausgegangen sei, der Kläger habe Kenntnis von dem Mangel gehabt. Ob der Geschäftsführer der Beklagten "glaubhaft" angegeben habe, seiner Auffassung nach enthielten die beiden zitierten Aufsätze nur abstrakte Ausführungen die Händescheidung zwischen Fohr und Rottmann, sei in diesem Zusammenhang irrelevant. Vielmehr komme es auf den objektiven Inhalt der Aufsätze an, der von den Angaben der Beklagten indes abweiche. So habe SV2 die streitgegenständliche Arbeit konkret Rottmann zugeschrieben, SV1 habe auf diese Ausführungen SV2s unmittelbar Bezug genommen. Auch aus dem Schreiben SV1s vom 27.6.2015 ergebe sich, dass er und SV2 sich konkret mit der Urheberschaft Rottmanns und der fehlenden Urheberschaft Fohrs an der "Bildtitel1" befasst hätten. Soweit es sich bei dem Motiv der "Bildtitel1" um ein bekanntes Motiv Carl Philipp Fohrs aus dessen „Skizzenbuch der Neckargegend" handele, sei es dem Kläger 2008 unbekannt gewesen, dass Rottmann 1812 „nach Fohr“ gezeichnet habe.

Überdies übergehe das Landgericht auch die schriftlichen Angaben SV1s, wonach 1997 das Museum1 Stadt2 sechs Zeichnungen Fohrs erlangt habe, von denen drei motivgleich mit Zeichnungen Rottmanns gewesen seien. Diese Neuentdeckungen habe SV1 ausführlich mit SV2 erörtert und in diesem Zusammenhang auch über die streitgegenständliche Zeichnung gesprochen. Das gemeinsame Urteil ergebe sich aus dem Aufsatz SV2s aus dem Katalog von 1998. SV1 selbst sei der Frage, ob Rottmann nach Fohr kopiert habe, oder ob beide Künstler vor demselben Motiv gesessen hätten, in seinem Aufsatz von 2001 nachgegangen. Auf die streitgegenständliche Zeichnung sei er dort nicht mehr eingegangen, weil ihm deren Zuordnung durch den Beitrag SV2s geklärt erschienen sei. Der Geschäftsführer der Beklagten habe aber genau gewusst, dass der Aufsatz SV2s mit dem SV1s in einem inneren Zusammenhang stehe und auf einem gemeinsamen Urteil beider Autoren beruht habe. Das habe er dem Kläger jedoch ebenso wenig offenbart wie den Umstand, dass die angeblich fehlerhafte Zuschreibung zu Rottmann gerade durch SV2 erfolgt sei. Zudem zähle es zu den Selbstverständlichkeiten wissenschaftlicher Arbeit, eine Zuordnung auf Basis neuer Erkenntnisse und Quellen zu revidieren, zumindest aber überprüfbar und transparent zu machen; das sei in dem Verkaufskatalog der Beklagten nicht geschehen.
2. Die Beklagte habe ferner auch ihre Pflicht zu ordnungsgemäßer Beratung des Klägers verletzt. Beratungspflichten würden nach ständiger Rechtsprechung des Bundesgerichtshofes dann begründet, wenn der Verkäufer im Rahmen eingehender Vertragsverhandlungen auf Frage des Käufers einen ausdrücklichen Rat erteile, oder wenn sich Erklärungen des Verkäufers nicht auf eine bloße Unterrichtung über Eigenschaften der Ware beschränkten, sondern der Käufer sich bei dem Verkäufer fachmännischen Rat einhole, so dass der Verkäufer die Stellung einer Vertrauensperson einnehme.

Im vorliegenden Fall ergebe sich eine Übernahme von Beratungspflichten schon aus dem Verkaufskatalog selbst, da die Beklagte auf eine angeblich fälschliche Zuweisung zu Rottmann in der Literatur hingewiesen habe und mit weiteren Literaturangaben eine Zuordnung zu Fohr habe belegen wollen. Hierdurch habe sie den Kläger vorsätzlich in die Irre geführt und getäuscht.

Zu Unrecht gehe das Landgericht davon aus, der Kläger habe seinerseits über ein erhebliches $M a ß$ an Sachkunde verfügt. Vielmehr habe dieser zum Zeitpunkt des Vertragsschlusses kein vertieftes Fachwissen besessen und seien ihm insbesondere die Aufsätze SV1s und SV2s unbekannt gewesen. Nähere Kenntnisse habe der Kläger vielmehr erst im Nachhinein durch aufwendige Recherchen erlangt.

Der Kläger beantragt,

1. die Beklagte zu verurteilen, an den Kläger $22.000,00 €$ nebst Zinsen in Höhe von 5 Prozentpunkten über dem jeweiligen Basiszinssatz p.a. seit dem 1.5.2014 zu zahlen,

Zug um Zug gegen Rückgabe der im Jahre 2008 gemäß "Rechnung/Lieferschein Nr. ..." als Werk Carl Philipp Fohrs verkauften Zeichnung „Bildtitel1", „Tuschfederzeichnung in Grauschwarz und Grau über Bleistift 1812", sowie

2. festzustellen, dass sich die Beklagte mit der Rücknahme der in Ziffer 1. näher bezeichneten Zeichnung in Annahmeverzug befindet.

\section{Die Beklagte beantragt,}

die Berufung zurückzuweisen.

Sie verteidigt das angefochtene Urteil unter Wiederholung und Vertiefung ihres erstinstanzlichen Vorbringens.

Insbesondere erläutere der Kläger nicht, woraus sich ergebe, dass der Geschäftsführer der Beklagten nicht in gutem Glauben gehandelt haben solle. Es sei nicht zu beanstanden, wenn das Landgericht darauf abstelle, dass die Beklagte den Rat von Fachleuten eingeholt habe; dies sei ein tauglicher Anknüpfungspunkt für den Rückschluss auf innere Tatsachen wie etwa das Vorliegen guten Glaubens. Soweit der Kläger eine Pflicht des 
Verkäufers annehme, in räumlicher, zeitlicher und inhaltlicher Hinsicht eine spezifizierende Aufklärung zu geben, lege er nicht dar, woraus sich diese Pflicht ergeben solle.

Weiter werfe der Kläger der Beklagten vor, einzelne Fundstellen unterdrückt zu haben. Über die Thematik Rottmann Fohr seien allerdings im Laufe der letzten mehr als 150 Jahre eine solche Vielzahl von Veröffentlichungen erschienen, dass auch ein Kunsthändler diese sicher nicht alle durchsehen, auswerten und präsentieren müsse. Ohnehin handele es sich bei jeder Stellungnahme nicht um die Bekundung einer objektiven Tatsache, sondern um das subjektive Urteil des jeweiligen Autors. Niemand könne mit absoluter Sicherheit behaupten, ob das streitgegenständliche Blatt von dem einen oder von dem anderen der beiden Künstler stamme; stets handele es sich nur um Zuschreibungen, Aussagen und Bewertungen, womit sich die Frage einer bewusst unrichtigen Behauptung oder eines arglistigen Verschweigens erledige. Im Übrigen bleibe es bei der Kennerschaft des Klägers und dessen beachtlicher Bibliothek zu Kunstwerken der deutschen Romantik.

Wegen des weiteren Sach- und Streitstands wird auf die in der Berufungsinstanz gewechselten Schriftsätze der Parteien verwiesen.

Der Senat hat die Parteien in der Sitzung vom 19.2.2016 persönlich angehört (BI. 357 ff. d.A.) und Beweis erhoben gemäß Beweisbeschlüssen vom 26.8.2016 (Bl. 416 f. d.A.) und 25.4.2017 (500 f. d.A.) durch Einholung eines Sachverständigengutachtens. Für das Ergebnis der Beweisaufnahme wird Bezug genommen auf die schriftlichen Gutachten des Sachverständigen SV3 vom 16.2.2017 (BI. 435 ff. d.A.) und 16.10.2017 (BI. 534 f. d.A.).

\section{III.}

Die Berufung ist zulässig und begründet.

1. Zu Unrecht hat das Landgericht einen dem Kläger gemäß $\S 346$ Abs. 1 BGB iVm §§ 437 Nr. 2, 434 Abs. 1 S. 2, 326 Abs. 5 BGB zustehenden Anspruch gegen die Beklagte auf Rückzahlung des Kaufpreises Zug um Zug gegen Rückgewähr der streitgegenständlichen Zeichnung verneint.

a) Die Zeichnung, die der Kläger aufgrund Kaufvertrags mit der Beklagten im Sommer 2008 erwarb und am 19.10.2008 übergeben erhielt, ist mangelhaft, weil sie entgegen der Katalogbeschreibung der Beklagten nicht der Hand Carl Philipp Fohrs zuzuschreiben ist.

aa) Ob die Klausel

Die Katalogbeschreibungen erfolgten nach bestem Wissen und Gewissen, sie sind keine Garantien im Rechtssinne. trotz $\S 305 c$ BGB überhaupt Vertragsbestandteil geworden ist, ihr angesichts der für die streitgegenständliche Zeichnung konkret verwendeten Katalogbeschreibung

Vgl. Carl Rottmann, Ausst.-Kat. Museum2 Stadt1, 1997/98, S. 12, Abb. 3 (dort fälschlich Carl Rottmann zugeschrieben).

der Vorrang der Individualabrede entgegensteht (vgl. BGH, Urteil vom 13.2.1980, VIII ZR 26/79, Rn. 24 - hier wie im Folgenden zitiert nach juris), sie lediglich eine Garantie im Sinne von $\S 443$ BGB ausschließen soll oder auch eine Beschaffenheitsvereinbarung im Sinne von $\S 434$ Abs. 1 S. 1 BGB und sie einer hiernach ggf. greifenden Inhaltskontrolle nach $\S \S 307$ ff. BGB standhielte, kann auf sich beruhen. Denn jedenfalls nicht berührt werden von der Klausel die Anforderungen an eine nicht vereinbarte Beschaffenheit im Sinne von $\S 434$ Abs. 1 S. 2 und 3 BGB. Das gilt bereits aufgrund des Wortlauts der Klausel, der für einen gegenteiligen Erklärungswillen der Beklagten keinen Anhalt gibt, jedenfalls aber aufgrund des aus $\S 305$ c Abs. 2 BGB folgenden Gebots kundenfreundlichster Auslegung (vgl. BGH, Urteil vom 9.10.2013, VIII ZR 224/12, Rn. 14).

bb) Die Echtheit eines Kunstwerks im Sinne seiner Herkunft aus der Hand eines konkreten Künstlers bestimmt maßgeblich die Eignung eines Kunstwerks als Sammlerstück und Wertanlage (vgl. BGH, Urteil vom 9.10.2013, VIII ZR 224/12, Rn. 13, vom 13.2.1980, VIII ZR 26/79, Rn. 29, und vom 15.1.1975, VIII ZR 80/73, $\mathrm{Rn} .13)$ und bildet daher regelmäßig dessen zentrale Eigenschaft für seine - im Rahmen eines Kaufvertrags der hier vorliegenden Art sowohl vorausgesetzte wie gewöhnliche - Verwendung (§ 434 Abs. 1 S. 2 Nr. 1 und 2 BGB).

Dieser rechtlichen Beurteilung steht nicht entgegen, dass es sich bei der vorliegenden Zeichnung um ein innerhalb der Fachwelt längere Zeit streitig zugeordnetes Kunstwerk handelt und gar nur einer bestimmten Stilepoche oder Malerklasse statt einem konkreten Künstler zuzurechnen wäre. Denn die seitens der Beklagten verwendete Katalogbeschreibung übt sich gerade nicht in Zurückhaltung der vorgenannten Art, sondern benennt einen konkreten Künstler und bezeichnet die alternativ in Betracht gezogene Urheberschaft Carl Rottmanns ausdrücklich als „fälschlich [...] zugeschrieben“.

cc) Nach dem gesamten Inhalt der Verhandlung und dem Ergebnis der Beweisaufnahme steht es zur freien Überzeugung des Senats fest (§ 286 Abs. 1 ZPO), dass "Bildtitel1" nicht der Hand Carl Philipp Fohrs entstammt.

Die richterliche Überzeugung erfordert auch im hier eröffneten Anwendungsbereich des § 286 ZPO keine - ohnehin nicht erreichbare - absolute oder unumstößliche, gleichsam mathematische Gewissheit, auch keine an Sicherheit grenzende Wahrscheinlichkeit, sondern nur einen für das praktische Leben brauchbaren Grad an Gewissheit, der verbleibenden Zweifeln Schweigen gebietet, ohne sie völlig auszuschließen (vgl. nur BGH, Urteil vom 18.10.2017, VIII ZR 32/16, Rn. 14; Zöller-Greger 
32. Aufl. 2018, § 286 Rn. 19). Unter Anlegung dieses Maßstabs besteht für den Senat kein vernünftiger Zweifel daran, dass die Zuordnung des Werks zu Carl Philipp Fohr unrichtig ist.

(1) Der Sachverständige schließt sich hinsichtlich der Einordnung der Zeichnung als stilistische Spätform - nämlich als ein eher in die 1790er Jahre zu datierendes, von einem empfindsamen ausklingenden Hollandismus im Sinne des endenden deutschen Louis-Seize-Stils bestimmtes Blatt -, die der Schülerschaft von (sehr) jungen Nachwuchskräften bei Friedrich Rottmann entstammt, der aus seiner Sicht plausiblen Einschätzung anderen Experten - namentlich SV4, SV5, SV2 und SV1 - an (BI. 436 d.A.). Hiermit stimme auch die auf der Zeichnung aufgebrachte Datierung "1812“ überein (BI. 435, 534 d.A.), die sich materialorientiert jedenfalls insoweit bestätigen ließe, als das verwendete Velinpapier in Deutschland erstmals 1783 Verwendung gefunden habe und zu Beginn des 19. Jahrhunderts dann von mehr Firmen produziert worden sei; darüber hinaus sei die Datierung „1812" unter Verwendung derselben Rußtusche geschrieben worden, die auch für die Zeichnung verwendet worden sei (Bl. 435 d.A.).

Eine nachvollziehbare Übereinstimmung mit dem Jugendwerk Carl Philipp Fohrs vermochte der Sachverständige demgegenüber in stilistischer Hinsicht überzeugend nicht zu erkennen. Für Fohr sei es kennzeichnend, dass er die überkommene akademische Zeichenweise origineller, differenzierter und innovativer, ästhetisch „sperriger" weiterentwickelt habe, so dass in grundlegender Weise typisch für ihn - auf die aus seiner Sicht prägnante Ausdrucksweise SV2s zurückgreifend - ein „Detailreichtum" sei, das auf einer "höchst variablen Linienführung" beruhe, im Unterschied zur "durchgehenden Schraffurtechnik" Carl Rottmanns. Auch bei der vorliegenden Zeichnung sei aber eine bloße Homogenität der Strichführung augenfällig, die gerade nicht vergleichbar mit Fohrs „höchst sensibler, ungemein detailreicher, mit feinsten Mischungen der Tusche arbeitenden Zeichenweise" sei, die bei seinen frühen Zeichnungen generell zu erkennen sei (BI. 436 f. d.A.).

Bei der Zeichnung handele es sich, wie aus der Zeichenweise folge, auch nicht um eine Kopie, sondern um eine Originalzeichnung, bei der der Künstler zunächst eine freie Vorzeichnung in Graphitstift vorgenommen und darüber, vergleichbar flüssig, in grauschwarzer Tusche mit Feder die Zeichnung ausgeführt habe - wobei im Vergleich von Vorzeichnung und Überzeichnung auch von ein und demselben Künstler auszugehen sei (BI. 435 d.A.).

Die gegen diese fundierte und wenngleich eher knapp, so doch prägnant und anhand gezielter Details begründete, insgesamt überzeugende Einschätzung vorgebrachten Einwände der Beklagten hat der Sachverständige stichhaltig zu entkräften vermocht. Insbesondere hat er zunächst die Kürze seiner stilistischen Einschätzung erläutert, die zum einen seinem Verweis auf die grundlegenden Beschreibungen durch SV2 geschuldet war, nicht zuletzt aber auch dem Umstand, dass „Kennerschaft bei Zuschreibungsfragen der vorliegenden Art sprachlich diffizil zu formulieren" und für Außenstehende - wie Juristen oft schwierig in "Argumentationsketten zu übersetzen" seien. Wenn die Beklagte daher meint, dass nach wie vor "das sprachliche Feuerwerk" des Sachverständigen nicht von einer "Stellungnahme zur eigentlichen Sachfrage begleitet" werde, geht dies an der inhaltlichen Auseinandersetzung mit den jeweiligen Stilbeschreibungen zu Fohr und Rottmann auf der Grundlage einer Inaugenscheinnahme der streitgegenständlichen Zeichnung und der hierbei festzustellenden Zeichenweise durch das kunsthistorisch geschulte Auge des Sachverständigen vorbei.

Soweit die Beklagte auf ein von ihr eingeholtes Schriftgutachten (BI. 487 ff. d.A.) verweist, wies der Sachverständige zutreffend darauf hin, dass der auf der Zeichnung befindliche Text „Bildtitel1. September 1812." ausweislich des Privatgutachtens zum einen schon gar nicht sicher Carl Philipp Fohr zuzuordnen sei, zum anderen aber auch ergänzend bemerkt, dass derartige Beschriftungen gar nicht stets durch den Urheber der jeweiligen Zeichnung erfolgt seien (Bl. 535 d.A.), weshalb für den Senat weder Existenz noch Inexistenz einer solchen Beschriftung höherer auch nur indizieller Beweiswert zukommt, der innerhalb der Beweiswürdigung gegenüber dem Zeichenstil durchgreifende Bedeutung beigemessen werden könnte.

Auch die weiteren, gegen die Sachkunde des Sachverständigen gerichteten Einwände greifen nicht durch. Wie durch diesen ergänzend dargelegt und von der Berufung im Nachgang hierzu auch nicht weiter angegriffen, wurde seine online erhältliche Publikationsliste seit einigen Jahren nicht mehr aktualisiert. Tatsächlich umfassten die Graphischen Sammlungen der ...stiftung Stadt3, die der Sachverständige betreue, aber auch Werke Stadt1 Romantiker und spezifisch auch solche von Carl Philipp Fohr, weshalb der Sachverständige zum stilkritischen Vergleich vor Ort auf altbekannte Bestände habe zurückgreifen können, die er entgegen den Einwänden der Beklagten nicht vollständig im Einzelnen aufzuführen hatte. Auf dieser Grundlage habe er überdies auch, unter Angabe der Fundstelle näher dargelegt, konkret zu Carl Philipp Fohr publiziert (BI. 534 d.A., Bl. 555 d.A.).

(2) Es kommt hinzu, dass es sich bei der Beurteilung durch den Sachverständigen, was ihre Überzeugungskraft eigenständig stützt, auch nicht etwa um eine singuläre Einschätzung handelt, der maßgebliche Forschung entgegenstünde, und die daher noch ausführlicherer Begründung bedürfte. Vielmehr verweist der Sachverständige im Gegenteil nachvollziehbar auf andere Stimmen der Fachliteratur, die seine Einschätzung sowohl im Hinblick auf das generelle stilistische Verhältnis Fohr - Rottmann teilen, wie auch im Hinblick auf die konkrete Herkunftszuordnung der "Bildtitel1".

So beschrieb in einem 1998 publizierten Beitrag (BI. 38 ff. d.A.) SV2 die Zeichenweise Rottmanns als stark von der Fohrs beeinflusst, wie u.a. die Zeichnung „Bildtitel1“ zeige - die bei SV2 als Abbildung 3 mit "Carl Rottmann, Bildtitel1, 1812" be- 
schrieben wird (BI. 40 f. d.A.). SV2 bezieht sich im Zusammenhang hiermit auf einen Beitrag SV5s, die - hinsichtlich einer anderen Zeichnung, jedoch übereinstimmend mit der vergleichenden Kennzeichnung des stilistischen Vermögens beider Künstler durch SV2 - von, gewissen Schwächen gegenüber der Fohr-Zeichnung' sprach, aufgrund derer sie vermutetet, dass es sich bei dieser anderen Zeichnung „um eine Kopie Rottmanns nach Fohr" handele (BI. 41 d.A.). Konkret wiederum bezogen auf die „Bildtitel1" macht SV2 dann „eine gröbere, ungelenkere und damit frühere zeichnerische Stufe" noch innerhalb des Jugendwerks Rottmanns selbst aus (BI. 42 d.A.). Dieses Gefälle im künstlerischen Vermögen beider Maler weiterhin betonend, bewertet SV2 denn auch die weitere Entwicklung in Rottmans Werk als eine Umdeutung von ,Fohrs höchst sensibler, ungemein detailreicher, mit feinsten Mischungen der Tusche arbeitenden Zeichenweise', bei der „Fohrs Detailreichtum“ verloren gehe (BI. 43 d.A.).

Nicht anders als SV2 beschrieb dann auch SV1 in einem 2001 erschienen Beitrag (BI. 19 ff. d.A.) Fohr als den begabteren der beiden Künstler, an dem sich Rottmann orientierte, dessen Strichbild gegenüber dem lebendigen Strichbild bei Fohr geradezu schematisch und starr anmute, während Fohr über eine differenzierte, in Richtung und Stärke wechselnde Federführung verfüge, die in einzelnen Motiven lebhaftes Licht- und Schattenspiel entstehen lasse, wo Rottmann mit schematisch fortfahrender Schraffur arbeite. Was die Einordnung konkret des streitgegenständlichen Werks betrifft, ist zwischen den Parteien darüber hinaus unstreitig, dass es schließlich auch durch SV1 Fohr zugeordnet wurde, auch wenn SV1 seine Einschätzung nicht publiziert, sondern schriftlich nur durch Schreiben an den Kläger vom 27.06.2015 (BI. 208 f. d.A.) geäußert hat, dies jedoch eigenen Angaben zufolge als Ergebnis intensiver Diskussionen mit SV2 bereits 1998, nachdem SV1 zunächst - etwa 1995 mündlich einen anderen Standpunkt vertreten hatte. Übereinstimmend hiermit gelangte schließlich denn auch SV6 zu der Einschätzung, dass es sich bei der "Bildtitel1" nicht um ein Werk Fohrs handelte.

Weshalb und auf welcher fachlichen Grundlage abweichend hiervon SV4 die Zuordnung der Zeichnung zu Rottmann bei SV2 für ein Versehen befunden haben soll, erschließt sich dem Senat nicht, muss mangels hierzu vorgetragener Anknüpfungstatsachen sowie des Umstands, dass SV4 verstorben ist, aber auch auf sich beruhen. Soweit die Beklagte darüber hinaus hinsichtlich der Einordnung der "Bildtitel1" als Zeichnung Fohrs auf SV7 verweist, hat der Sachverständige - im Nachgang unwidersprochen - klargestellt, dass die entsprechende Textpassage im Katalog "Natur als Kunst“ von 2013 (BI. 216 d.A.) nicht von SV7, sondern von SV8 stammt, der zwar eine Zuordnung der „Bildtitel1“ zu Fohr vornimmt, dies jedoch lediglich auf der Grundlage des Katalogs der Beklagten von 2008.
Bestanden bei dieser Sachlage aber insgesamt keine Zweifel an dem Urteil des Sachverständigen, war weder die Einholung eines ergänzenden oder eines weiteren Gutachtens geboten noch auch nur die mündliche Anhörung des Sachverständigen - die denn auch seitens der Parteien nicht beantragt wurde.

b) Einer Fristsetzung zur ordnungsgemäßen Erfüllung des abgeschlossenen Kaufvertrages bedurfte es nicht, da Übergabe und Übereignung der vorliegenden Zeichnung als einer solchen von Carl Philipp Fohr bereits bei Vertragsschluss unmöglich war, §§ 437 Nr. 2, 326 Abs. 5, 275 Abs. 1 BGB.

c) Einem Rücktritt des Klägers stand eine Verjährung seiner Mängelansprüche nicht entgegen, da sich diese im vorliegenden Fall nach $\S 438$ Abs. 3 S. 1 BGB richtet und die deshalb gemäß $§ 195$ BGB drei Jahre betragende Verjährungsfrist gemäß $\S 199$ Abs. 1 BGB erst mit Schluss des Jahres 2011 zu laufen begann, aufgrund am 19.12.2014 unter Gerichtskostenvorschusszahlung eingereichter, aufgrund Verfügung vom 13.1.2018 am 22.1.2015 und damit "demnächst" im Sinne von § 167 ZPO zugestellter Klage jedoch in ihrem Ablauf gemäß § 204 Abs. 1 Nr. 1 BGB gehemmt wurde.

aa) Die Beklagte muss sich hinsichtlich der unrichtigen $\mathrm{Zu}$ ordnung der Zeichnung zu Fohr arglistiges Handeln im Rechtssinne vorhalten lassen ( $\$ 438$ Abs. 3 S. 1 BGB), das nicht erst bei betrügerischer Vorgehensweise vorliegt, sondern bereits dann, wenn der Erklärende die Unrichtigkeit seiner Erklärung für möglich hält und an dieser dennoch ohne Anmeldung eines Vorbehalts festhält, die Unrichtigkeit seiner Äußerung mithin billigend in Kauf nimmt.

Zwar handelt mangels Vorsatzes grundsätzlich, wie die Berufung im Ausgangspunkt zutreffend ausführt, nicht arglistig, wer gutgläubig unrichtige Angaben macht, mag auch der gute Glaube auf Fahrlässigkeit oder selbst auf Leichtfertigkeit beruhen. Zur Arglist ist umgekehrt aber auch nicht notwendig das Wissen erforderlich, dass die angegebene Tatsache nicht der Wahrheit entspricht. Arglist ist vielmehr schon dann anzunehmen, wenn der Verkäufer ohne tatsächliche Grundlage unrichtige Angaben über die Mängelfreiheit oder über wesentliche Eigenschaften der Kaufsache macht, die geeignet sind, den Kaufentschluss des Käufers mit zu beeinflussen (vgl. insbesondere BGH, Urteil vom 18.3.1981, VIII ZR 44/80, Rn. 13, vom 27.9.2017, VIII ZR 271/16, Rn. 46, und vom 22.4.2016, 22.04.2016, V ZR 23/15, Rn. 21 f.). Denn in diesem Fall erübrigt sich aus der Sicht des Käufers eine weitere Überprüfung, weil er davon ausgehen darf, dass der Verkäufer seine Erklärungen nicht „ins Blaue hinein“ abgibt. Der die Arglist begründende Vorwurf gegenüber dem Verkäufer liegt in einem solchen Fall mithin in dem Umstand, dass der Erklärende, obschon ihm bewusst ist, dass ihm die zur sachgemäßen Beantwortung erforderliche Kenntnis fehlt, diesen Umstand gleichwohl gegenüber dem anderen Teil verschweigt (vgl. BGH, Urteil vom 18.3.1981, VIII ZR 44/80, Rn. 14). 
Eben dies muss sich die Beklagte entgegenhalten lassen. Ihr Geschäftsführer hat eingeräumt, dass ihm die Zuordnung zu Rottmann durch SV2, wie die Angaben im Verkaufskatalog auch bestätigen, bekannt war. Ebenso war dem Geschäftsführer der Beklagten bekannt, dass es sich bei SV2 um einen Fohr-Experten handelte. Dann aber durfte die Beklagte auf der Grundlage ihres eigenen Vorbringens und der ihr danach zur Verfügung stehenden weiteren (mündlichen) Expertisen dessen Auffassung nicht apodiktisch als falsch darstellen und gegenüber dem Leser ihres Katalogs den hierdurch vermittelten Anspruch erheben, das Werk kraft vorhandener Sachkunde zweifelsfrei Fohr zuschreiben zu können.

Bei den Mitteilungen des für den einreichenden Eigentümer handelnden Kunsthändlers A über die Begutachtung des Blattes durch SV1 und SV2 handelte es sich, wie die Beklagte wusste, um bloße Begutachtungen vom Hörensagen, die von vornherein nur sehr eingeschränkt belastbar waren und von ihr gleichwohl keine adäquaten Überprüfung zugeführt wurden - obschon dies angesichts des danach bestehenden Widerspruchs in der Zuschreibung durch SV2 ersichtlich geboten war. Zugleich sprachen die potentielle Fehlerträchtigkeit einer Weitergabe mündlicher Informationen sowie ein zumindest in Betracht zu ziehendes Eigeninteresse des Eigentümers zusätzlich für die Notwendigkeit einer adäquaten Überprüfung.

Von Relevanz für die Beklagte konnten daher allein ihre eigene Expertise bzw. diejenige der Kunsthistoriker SV4, SV5 und SV7 - mit Rücksicht auf die Ausführungen des Sachverständigen gemeint wohl SV8, mit dem die Beklagte allerdings gesprochen zu haben selbst nicht behauptet - sein, was aber nichts daran ändert, dass rein mündliche Expertisen aus den dargelegten Gründen ersichtlich unzureichende Grundlagen für die Katalogbeschreibung der Beklagten waren, die die Urheberschaft Fohrs demgegenüber aber nicht nur als "möglich" oder "wahrscheinlich" bezeichnete, sondern als völlig fraglos darstellte. Dass es sich bei der publizierten Zuordnung zu Rottmann durch SV2 gar um ein unplausibles Fehlurteil handelte, wie die Beklagte mit der SV4 zugeschriebenen Formulierung "Versehen" Glauben machen will, ist gerade nicht ersichtlich; vielmehr bezieht sich auch der Sachverständige weiterhin auf dessen Einschätzungen als bis heute "grundlegend“.

Der Senat verkennt bei dieser Beurteilung nicht, dass ein Kunsthändler hinsichtlich der Echtheit der von ihm angebotenen Kunstwerke typischerweise ein erhebliches Risiko trifft, weil er regelmäßig schon angesichts eines häufigen Eigentumswechsels gar nicht in der Lage ist, durch zumutbare eigene Nachforschungen Sicherheit über die Echtheit des Werks zu erlangen (vgl. bereits BGH, Urteil vom 15.1.1975, VIII ZR 80/73, Rn. 15; ferner BGH, Urteil vom 13.2.1980, VIII ZR 26/79, Rn. 20 ff.). Dass weitergehende Nachforschungen auch im vorliegenden nicht zumutbar gewesen seien, behauptet die Beklagte indes selbst nicht; ihr eigenes Verhalten vor Erstellung des Katalogs spricht denn auch dagegen. Entscheidend ist jedoch, dass selbst derjenige, der keine hinlängliche Gewissheit haben kann, eine solche Gewissheit gegenüber seinen Kaufinteressenten auch nicht vorgeben darf. Eben dies hat die Beklagte jedoch mit ihrer apodiktischen Formulierung "dort fälschlich Carl Rottmann zugeschrieben" getan und dies mit ihrem allgemeinen Hinweis, ihre Katalogbeschreibung "nach bestem Wissen und Gewissen“ erstellt zu haben, zusätzlich verstärkt.

bb) Begründete Zweifel des Klägers an der Zuordnung zu Fohr erlangte der Kläger frühestens im Dezember 2011 aufgrund der Einschätzung von SV1, das Blatt stamme eher von Rottmann, so dass die Verjährung frühestens mit Ablauf dieses Jahres einsetzte. Eine vorherige Kenntnis oder grob fahrlässige Unkenntnis des Klägers ist weder nachvollziehbar vorgetragen noch ersichtlich, weshalb Ansprüche des Klägers zugleich nicht an $\S 442$ Abs. 1 BGB scheitern. Insbesondere musste die Angabe im Verkaufskatalog der Beklagten den Kläger nicht veranlassen, nähere Erkundigungen zur Urheberschaft einzuholen. Denn im Katalog war die Zuschreibung zu Rottmann für einen Leser eindeutig als „fälschlich“ deklariert worden.

Erhebliches Fachwissen des Klägers hat die Beklagte zwar behauptet, aber weder substantiiert dargetan noch auch nur konkrete Anknüpfungstatsachen benannt, aufgrund derer der Kläger zu einer vergleichbaren wissenschaftlichen Einschätzung hätten gelangen können bzw. auch nur die Fehlerhaftigkeit der - kunsthistorische Absicherung in Anspruch nehmenden - Katalogbeschreibung hätte erkennen müssen. Die vage gehaltene Formulierung, der Kläger besitze eine ,beachtliche Bibliothek zu Kunstwerken der deutschen Romantik', reduziert sich bei dieser Sachlage auf eine bloße Vermutung 2008 bereits vorhandenen Fachwissens, die zudem allgemein gehalten bleibt und sich nicht auf die konkrete Zeichenweise von Fohr einerseits und Rottmann andererseits bezieht.

2. Die mit der Klage verfolgten Zinsen waren dem Kläger unter dem Gesichtspunkt des Verzugs zuzusprechen, §§ 280 Abs. 1 u. 2, 286 BGB; sein mit Rücksicht auf $\S 756$ Abs. 1 ZPO zulässiger Feststellungsantrag ist aus den vorstehenden Gründen und angesichts der durch Schreiben vom 28.4.2014 konkret angebotenen Rückgabe der Zeichnung ebenfalls begründet.

\section{IV.}

Die Entscheidung über die vorläufige Vollstreckbarkeit folgt aus $\S \S 708$ Nr. 10 S. 1, 711 ZPO, die Festsetzung des Streitwerts aus $\S 47$ Abs. 1 S. 1 GKG.

Die Revision war nicht zuzulassen, da die Voraussetzungen des § 543 Abs. 2 S. 1 ZPO nicht vorliegen; die Rechtssache hat weder grundsätzliche Bedeutung, noch erfordert die Fortbildung des Rechts oder die Sicherung einer einheitlichen Rechtsprechung eine Entscheidung des Revisionsgerichts. 